\title{
Performance Analysis of MIMO Assisted Interleave Division Multiple Access System with Multi-user Detection
}

\author{
Vishvaksenan, K.S. \\ Department of Electronics and \\ communication Engineering \\ SSN College of Engineering \\ Chennai-India
}

\author{
Seshasayanan .R \\ Department of Information and \\ communication Engineering \\ Anna University \\ Chennai-India
}

\begin{abstract}
This paper presents the performance analysis of Multi Input Multi Output (MIMO) assisted interleave based multiple-access system. In IDMA, different interleavers are used to distinguish users as against different signature sequence in a conventional code-division multiple-access (CDMA) scheme. The basic principle of IDMA is that the interleaver is unique for the users. In this work, we consider that Interleavers are generated independently and randomly. .Also the IDMA technique is extended to multi user MIMO IDMA with multiuser detection. At the receiver, SIC detector is employed. The performance of the system is analyzed for different channel conditions using extensive simulation runs based on Monte Carlo simulation trials. It is shown that the IDMA scheme can achieve near single user performance in situations with very large numbers of users while maintaining very low receiver complexity. Simulation examples are provided to illustrate the advantages of the IDMA.
\end{abstract}

Keywords: CDMA, channel capacity, iterative decoding, multi-user detection

\section{INTRODUCTION}

Code division multiple access system is the most widely used system for multiuser communications. But the performance of CDMA is limited by multiple access interference and intersymbol interference. With CDMA fading is circumvented by the use of interleavers placed between FEC and spreading. After the invention of joint Turbo type receivers, extensive studies have been made to mitigate MAI and ISI employing joint detection and decoding. But high complexity of optimal detection precludes its implementation for signal detection. he interleavers, which are primarily implemented for combating fading in CDMA system, can be employed to distinguished user signals. This principle has been investigated [1], and its potential merits have been addressed .This system in which interleavers are employed as means of user separation is called IDMA.

IDMA retains several advantages of CDMA like a asynchronous transmission, Cross cell interference and robustness against fading.

In interleave division multiple access system, user specific interleavers are employed. IDMA has been demonstrated to outperform CDMA with multiuser detection [1][2].

Theoretical analysis has shown that optimal capacity of multiple access scheme can be achieved when the entire bandwidth is utilized for coding .In IDMA, user specific interleavers combine with low rate channel coding is used for user seperation. Here the interleavers are generated randomly and independantly. It is a wide band system and multiuser detection with moderate complexity can be used for signal detection.

Multi input and multi output (MIMO) system has proved in the recent past to provide very high capacity [1] without any increase in the transmission bandwidth and power. The informationtheoretic capacity of these multiple-input multiple-output channels was shown to grow linearly with smaller numbers of transmit and receive antennas in rich scattering environments, and at sufficiently high signal-to-noise (SNR) ratios [4][5] [6]. MIMO profile in any wireless communication system can be realized by two schemes namely, 1) using the classical BELL labs architecture (VBLAST-Vertical Bell Laboratories Space Time architecture) and 2) Space Time Block Codes. So combining Vblast architecture with the IDMA system can result in MIMO- IDMA that can offer bandwidth efficiency, space multiplexing and lower speed parallel type of signal processing and interference rejection capability (ISI reduction) in high datarate transmission. Of late, significant progress has been made in multi-user detection for code-division multiple-access (CDMA) systems [7]. In this paper, we investigate the performance of the MIMO assisted interleave division multiple access (MIMOIDMA) scheme with multiuser detection. We will show that the MIMO IDMA scheme can provide better performance with the aid of VBLAST/ZF [1] detection technique.

Also this approach is independent of the number of users and gives better performance in multipath environment. The rest of the paper is organized as follows- the transmitter and receiver structures for CDMA and IDMA is presented in section 2. System model is presented in section 3. Section 4 gives the essence of signal detection techniques. Estimation technique is presented in Section 5.Sectin 6 illustrates chip by chip detection Performance results will be presented in section 7 . Eventually conclusions are drawn in section 8 .

\section{TRANSMITTER AND RECEIVER STRUCTURES FOR CDMA AND IDMA}

Conventional DS-CDMA 


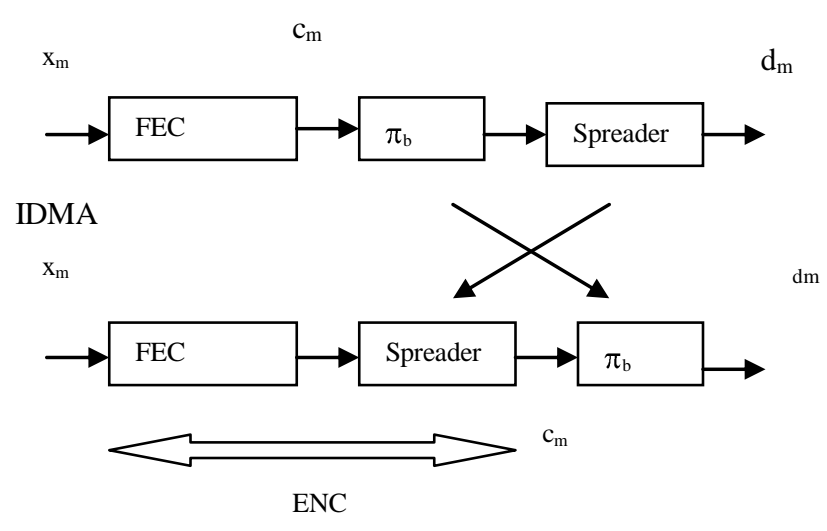

Fig. 1. Comparison of conventional DS-CDMA with IDMA. The data sequence of the m-th layer is denoted as $\mathrm{xm}$ and the corresponding encoded and interleaved sequence is denoted as dm.

The use of signature sequences for user separation is a characteristic feature for a conventional CDMA system. Interleaving, which is usually placed between forward error correction (FEC) coding and spreading, is employed to combat fading effect.

DS-CDMA is a popular transmission technique already applied in 2G (IS-95), 2.5G (cdma-2000), and 3G (UTRA, FDD, UTRA TDD, TD-CDMA) systems. Distinct data streams $\mathrm{dm}$ are distinguished by different spreading sequences. Along with channel coding, forward error correction (FEC) coding is typically done before interleaving and spreading, as shown in the upper part of Fig. 2. Typically, the same FEC encoder and the same interleaver are used for all data streams $\mathrm{dm}$.

In Fig. 2, a DS-CDMA system is illustrated where the arrangement of interleaving and spreading is reversed. Now, different interleavers distinguish distinct data streams. This special case of DS-CDMA is called code-spread CDMA, chipinterleaved CDMA (cI-CDMA), or interleave-division multiple access (IDMA) in the literature

In IDMA, a single low-rate encoder, subsequently denoted by ENC, may do FEC encoding and spreading jointly. The spreader has no special function. Furthermore, it is important to note that interleaving is done on a chip-by-chip basis.

\section{SYSTEM MODEL}

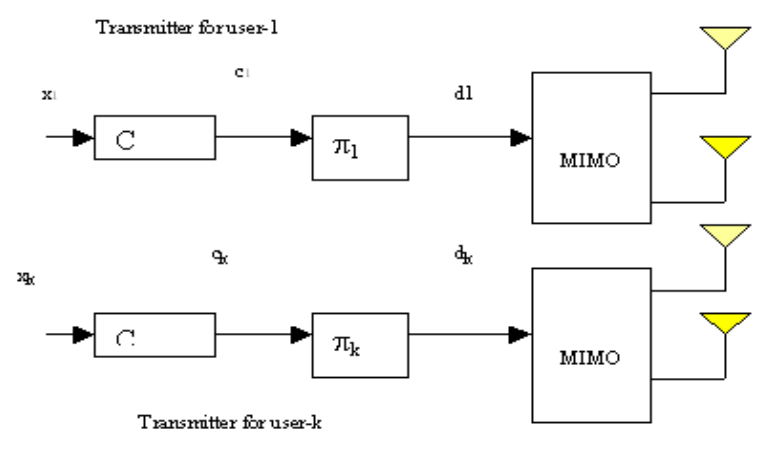

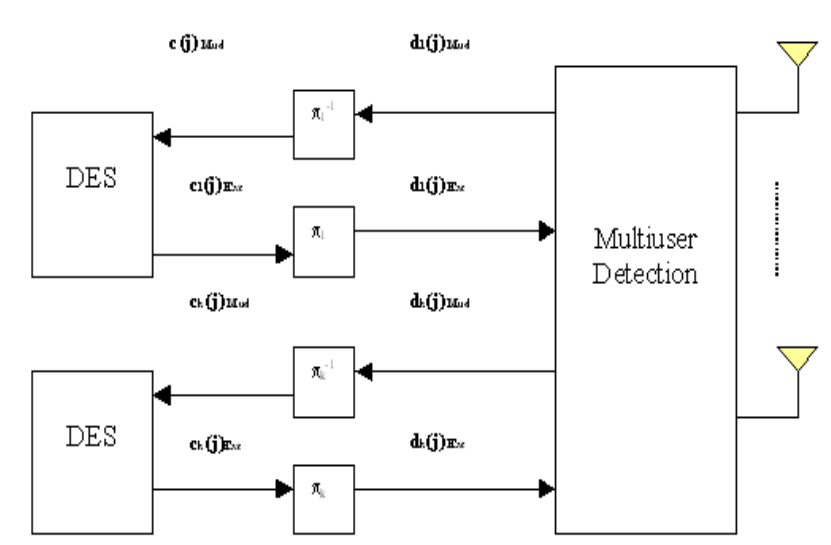

Fig 2. Transmitter and (iterative) receiver structures of an IDMA-MIMO scheme with $\mathrm{K}$ simultaneous users and V-Blast architecture.

The upper part of Fig 3 shows the transmitter structure of the multiple access schemes under consideration with $\mathrm{K}$ simultaneous users and V-Blast architecture. The input data sequence $\mathrm{x}_{\mathrm{k}}$ of user-k is encoded based on a low-rate code $\mathrm{S}$, generating a coded sequence $c_{k}=\left[c_{k}(1), \ldots, c_{k}(j), \ldots, c_{k}(J)\right]$, where $J$ is the frame length. The elements in ck are referred to as coded bits. Then ck is permutated by an interleaver pk, producing $d_{k}=\left[d_{k}(1), \ldots, d_{k}(j)\right.$, $\left.\ldots, \mathrm{d}_{\mathrm{k}}(\mathrm{J})\right]$. Following the CDMA convention, we call the elements in dk "chips". The interleavers solely distinguish users, hence the name interleave-division multiple-access (IDMA). In this paper we consider a multiuser MIMO IDMA system. Each of the synchronous user is equipped with $M$ transmit antennas and they communicate to a single base station, equipped with $N$ receive antennas. MIMO profile is realized by using VBLAST (Vertical Bell Laboratories Space Time architecture).

\section{SYMBOL DETECTION TECHNIQUE}

Considering a synchronous signal over a time variant channel, the received signal at the time instant $j$ is given as

$$
r(j)=\sum_{k=1}^{K} h_{k} d_{k}(j)+n(j), j=1,2, . . J
$$

where $H_{k}$ is the channel co-efficient for user $k$ and $\{n(j)\}$ are samples of an AWGN process with variance $\sigma^{2}=N o / 2$. Here perfect knowledge of the channel state information is assumed to be available at the receiver. In each use of the MIMO channel, a vector $\mathrm{d}=\left(\mathrm{d}_{1}, d_{2} \ldots d_{M}\right)^{T}$ of complex numbers is sent and a vector $\mathrm{r}=\left(r_{1}, r_{2}, \ldots \ldots, r_{N}\right)^{T}$ of complex numbers is received. We assume an input-output relationship of the form

$$
\boldsymbol{r}=\boldsymbol{H} \boldsymbol{d}+\boldsymbol{n}
$$

where, $\boldsymbol{H}$ is a $N$ x $M$ matrix representing the scattering effects of the channel and $n=\left(n_{1}, n_{2}, \ldots \ldots, n_{N}\right)^{T}$ is the noise vector. Throughout, we assume that $H$ is a random matrix with 
independent complex Gaussian elements $\left\{h_{i j}\right\}$ with mean 0 and unit variance, denoted by $h_{i j} \sim C N(0 ; 1)$. We also assume throughout that $n$ is identical and independent distributed (i.i.d) complex Gaussian random vector. The symbol detection problem considered in this paper is the problem of estimating the MIMO channel input vector $\boldsymbol{x}$ given the received vector $r$ assuming that the receiver has perfect knowledge of $\boldsymbol{H}$. The decision is made on a symbol-by-symbol basis. The next section discusses the VBLAST detector employed in our work.

\section{Zero-Forcing (ZF) Receiver}

Zero-Forcing $(\mathrm{ZF})$ receiver is a low-complexity linear detection algorithm that outputs,

$$
d=Q\left(\hat{d}_{Z F}\right)
$$

where

$$
\hat{d}_{Z F}=H^{+} r
$$

and $H^{+}$denotes the Moore-Penrose pseudo inverse of $H$, which is a generalized inverse that exists even when $H$ is rank-deficient.

\section{V-BLAST Detection}

For user-k, the corresponding ESE outputs $\left\{\mathrm{L}\left(\mathrm{d}_{\mathrm{k}}(\mathrm{j})\right), \mathrm{j}=1,2, \ldots \mathrm{J}\right\}$ are de-interleaved to form $\left\{\mathrm{L}\left(\mathrm{d}_{\mathrm{k}}(\mathrm{j})\right), \mathrm{j}=1,2, \ldots \mathrm{J}\right\}$ and delivered to the DES for user-k. The DES performs a soft-in/soft-out chip-by-chip de-spreading operation as detailed below. For simplicity, we focus on the chips related to $\mathrm{d} 1(\mathrm{k})$, the first bit of user-k. The treatment for other chips is similar

The V-BLAST detection algorithm [6] is a recursive procedure that extracts the components of the transmitted vector $d$ according to a certain ordering $\left(k_{1}, k_{2} \ldots k_{M}\right)$ of the elements of $\mathrm{d}$, where, $\left(k_{1}, k_{2} \ldots k_{M}\right)$ is a permutation of $(1 \ldots M)$. In VBLAST, this permutation depends on $\boldsymbol{H}$ (which is known at the receiver by assumption) but not on the received vector $\mathbf{r}$. The VBLAST/ZF algorithm is a variant of VBLAST derived from ZF rule. The algorithm determines the order of layers to be detected performs nulling and computes the decision statistics. It then slices the computed decision statistics and yields the decision by performing cancellation with the help of decision feedback, and finally computes the new pseudo-inverse for the next iteration. V-BLAST/ZF may be seen as a successive-cancellation scheme derived from the $\mathrm{ZF}$ scheme discussed in the previous section. The ZF rule creates a set of sub channels by forming

$$
\hat{d}=\left(\boldsymbol{H}^{+} \boldsymbol{H}\right) \boldsymbol{d}+\boldsymbol{H}^{+} \boldsymbol{n}
$$

The $j^{\text {th }}$ such sub-channel has noise variance $\left\|\left(\mathrm{H}^{+}\right)_{j}\right\|^{2} N_{0}$ and the order selection rule prioritizes the sub-channel with the smallest noise variance.

\section{ESTIMATION}

The ESE outputs are the logarithm likelihood ratios (LLRs) [9]about $\left\{d_{k}(j)\right\}$ computed based on (3) as

$$
\begin{aligned}
L\left(d_{k}(j)\right) & \equiv \log \left[\frac{\operatorname{Pr}\left(d_{k}(j)=+1 \mid r_{j}\right)}{\operatorname{Pr}\left(d_{k}(j)=-1 \mid r_{j}\right)}\right] \\
= & \frac{2 h_{k}\left(r(j)-E\left(\eta_{k}(j)\right)\right.}{\operatorname{Var}\left(\eta_{k}(j)\right)}, \forall k, j
\end{aligned}
$$

Where $r(j)=h_{k} d_{k}(j)+\eta_{k}(j)$

$$
\begin{aligned}
& E\left(\eta_{k}(j)\right)=E(r(j))-h_{k} E\left(d_{k}(j)\right) \text { and } \\
& \operatorname{Var}\left(\eta_{k}(j)\right)=\operatorname{Var}(r(j))-\left|h_{k}\right|^{2} \operatorname{Var}\left(d_{k}(j)\right)
\end{aligned}
$$

\section{CHIP BY CHIP DETECTION}

The a posteriori LLR for $\mathrm{x}_{\mathrm{k}}(\mathrm{j})$ can be computed using $\left\{\mathrm{L}_{\mathrm{k}}(\mathrm{c}(\mathrm{j}))\right\}$ as

$$
\begin{aligned}
L\left(x_{k}(j)\right) & \equiv \log \left[\frac{\operatorname{Pr}\left(x_{k}(j)=+1 \mid r\right)}{\operatorname{Pr}\left(x_{k}(j)=-1 \mid r\right)}\right] \\
& =\sum_{j=1}^{S} s_{k}(j) L\left(c_{k}(j)\right)
\end{aligned}
$$

The extrinsic LLR for a chip cj(k) within $\mathrm{d} 1(\mathrm{k}) \mathrm{s}(\mathrm{k})$ is defined by

$$
\left(c_{k}(j)\right)_{E x t} \equiv \log \left[\frac{\operatorname{Pr}\left(c_{k}(j)=+1 \mid r\right)}{\operatorname{Pr}\left(c_{k}(j)=-1 \mid r\right)}\right]-L\left(c_{k}(j)\right)
$$

The extrinsic LLRs $\left\{\left(\mathrm{c}_{\mathrm{k}}(\mathrm{j})_{\mathrm{Ext}}\right)\right\}$ form the outputs of the DES and are fed back to the ESE after interleaving. In the next iteration, $\left\{\operatorname{Ext}\left(\mathrm{d}_{\mathrm{k}}(\mathrm{j})\right)\right\}$ are used to update $\left\{\mathrm{E}\left(\mathrm{d}_{\mathrm{k}}(\mathrm{j})\right)\right\}$ and $\left.\left\{\operatorname{var} \mathrm{d}_{\mathrm{k}}(\mathrm{j})\right)\right\}$ as

$$
E\left(d_{k}(j)\right)=\frac{\exp \left(\left(d_{k}(j)\right)_{E x t}\right)-1}{\exp \left(\operatorname{Ext}\left(d_{k}(j)\right)\right)+1}=\tanh \left[\frac{\left(d_{k}(j)\right)_{E x t}}{2}\right]
$$

$\operatorname{Var}\left(d_{k}(j)\right)=1-E\left(d_{k}(j)\right)^{2}$ 


\section{SIMULATION RESULTS}

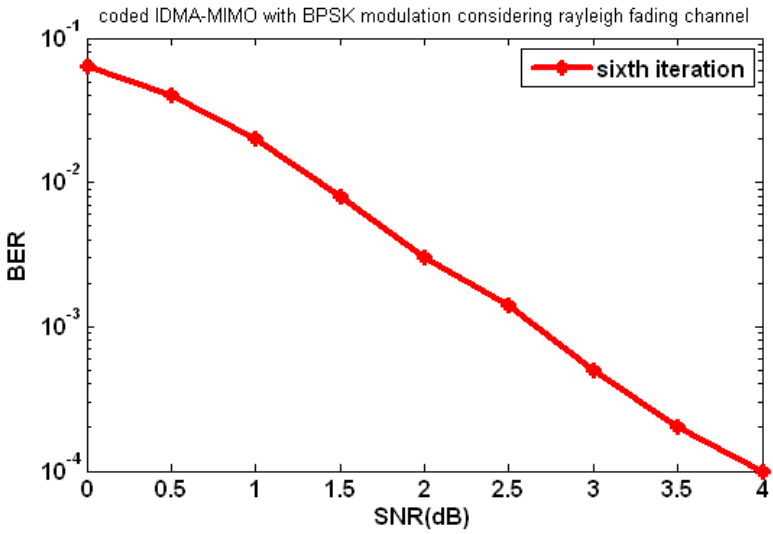

Fig.3. Bit error rates (BER) of IDMA VBLAST/ZF with 50 users for quasi-static Rayleigh fading channel after $6^{\text {th }}$ iteration

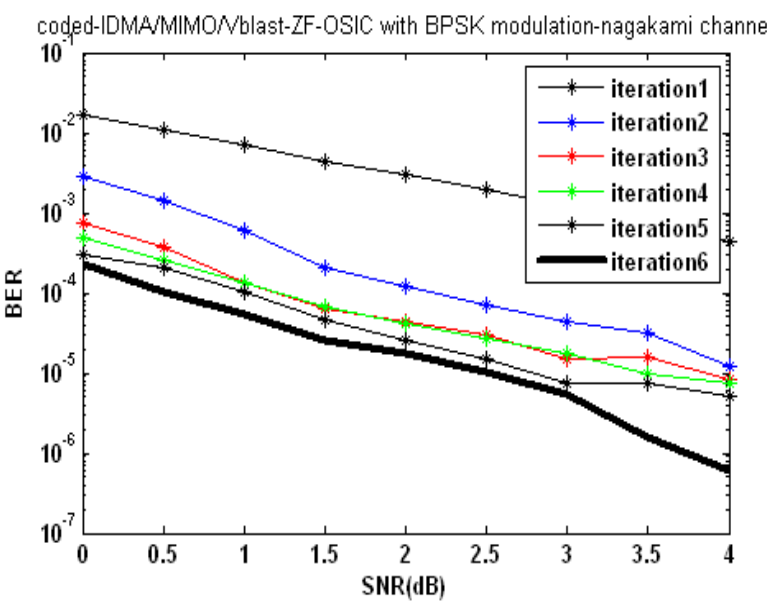

Fig.4. Bit error rates (BER) of IDMA VBLAST/ZF with 50 users for nakagami channel for 6 iteration

Fig 3 expounds the simulation results for an $2 \times 2$ MIMO IDMA system employing VBLAST/ZF detectors considering Raleigh fading channel with 50 user. Fig 4 evince the simulation results for an $2 \times 2$ MIMO /IDMA system employing VBLAST/ZF detectors with nakagami channel with 50 user The modulation technique considered is BPSK and the $E_{b} / N_{0}$ ranges between $-0 \mathrm{~dB}$ and $5 \mathrm{~dB}$. The channel matrix $\mathrm{H}$ is a random matrix with independent complex Gaussian elements $\left\{h_{i j}\right\}$ with mean 0 and unit variance The Bit error rate BER is calculated by performing 10,000 trials at each $E_{b} / N_{0}$ point..

\section{CONCLUSIONS}

In this paper we have considered multiple access scheme for $4 \mathrm{G}$ mobile communications and spatially multiplexing. Efficient transmission and symbol detection in the presence of multi stream interference and multiple access interference are presented. The benefits of IDMA scheme with specially multiplexing are substantial as seen from the fig4-5 that it supports many users with less complexity of receiver in multipath environment

\section{REFERENCES}

[1] Clemens Novak,Franz Hlawatch, and Gerald Matz,"MIMOIDMA: Uplink Multiuser MIMO Communications using interleave Division Multiple access and Low -Complexity Iterative Receivers",IEEE ICASSP 2007

[2] Li Ping, L. Liu, K. Y. Wu, and W. K. Leung, "Interleavedivision multiple-access (IDMA) communications," in Proc. 3rd International Symposium on Turbo Codes \& Related Topics, 2003, pp. 173-180

[3] Hendrik Schoeneich, Peter A. Hoeher, "Adaptive InterleaveDivisionMultiple Access -A potential air interface for $4 \mathrm{G}$ Bearer Services and Wireless LANs,” ICC, 2004.

[4] Prabagarane.N, C.S.Nivedita, Padmini S, Vijay C," Interleave Division Multiple Access System with iterative Multiuser Detection," IEEE ICACC, Feb 2007.

[5] Prabagarane.N,Ramakrishnan.M, Divya.T, Maria.Thomas, Jalaja.B, "Perfo-rmance Evaluation of Multi Stage Receivers for Coded Signals in MIMO Channels," 4th IEEE WiCOM, Oct 2008.

[6] I .E. Telatar, "Capacity of multi-antenna Gaussian channels," Eur. Trans. Tel., vol. 10, pp. 585-595, Nov-Dec 1999.

[7] Prabaagarane Nagaradjane, Arvind Sai Sarathi Vasan, Lakshmi Krishnan, "A Robust Space Time Co-Channel Interference Mitigation and Detection Technique for Multiuser MIMO Multicarrier DS/CDMA Systems", proc. IEEE International Conference, Wireless Vitae 2009.

[8] Prabaagarane Nagaradjane, Arvind Sai Sarathi Vasan, Lakshmi Krishnan,Anand Venkataswamy "Joint VBLAST/STBC Assisted MC DS/CDMA System with Multiuser Detection", proc. IEEE International Conference, WiCOM, Oct 2009.

[9] L.Ping, On "Interleave-division Multiple-Access", proc. IEEE ICC 04, Paris, June 2004, pp. 2869-73. 